\title{
DEVELOPMENT OF RESONANCE DEPOLARIZATION METHOD AT VEPP-4 FOR HIGH PRECISION MEASUREMENT OF TAU LEPTON MASS*
}

\author{
V.E. Blinov, A.V. Bogomyagkov, S.E. Karnaev, V.A. Kiselev, E.B. Levichev, \\ S.I. Mishnev, S.A. Nikitin ${ }^{\dagger}$, I.B. Nikolaev, D.M. Nikolenko, I.Ya. Protopopov, \\ Yu.A. Tikhonov, D.K. Toporkov, G.M. Tumaikin, V.N. Zhilich, N.I. Zinevich \\ BINP SB RAS, Novosibirsk, 630090, Russia
}

\begin{abstract}
We plan to measure the mass of $\tau$ lepton near the threshold of its production with an accuracy $10^{-4}$ or better in the experiment with the detector KEDR [1] at the e+e- collider VEPP-4M [2] using the method of resonance depolarization for calibration of particle energy. The scenario of experiment, the developed systems of polarimeters and the depolarizer are described. Some points of the beam polarization and the energy calibration accuracy are considered.
\end{abstract}

\section{INTRODUCTION}

The PDG [3] value of the $\tau$ lepton mass is $1777.03_{-0.26}^{+0.30}$ $\mathrm{MeV}$. The error given is based mainly on the measurement of the BES detector at the $e^{+} e^{-}$collider BEPC [4] where the value of the $\tau$ mass $1776.96_{-0.21-0.17}^{+0.18-0.25} \mathrm{MeV}$ was obtained near the threshold of $\tau$ production. The absolute energy scale and energy spread at the BEPC were determined by interpolation between results of repeated scans of the $J / \Psi$ and $\Psi^{\prime}$ resonances. The values of $J / \Psi$ and $\Psi^{\prime}$ masses from PDG were used. Inaccurate khowledge of the absolute value of beam energy in the vicinity of the threshold gives a main systematic error of this experiment. Theory predicts a quite simple relation of the tau mass with the lifetime and electronic branching fractions. Raising of an accuracy in determination of two last-named parameters is known to be planned in experiments at the $B$-factories. In conjunction with our planned result for the $\tau$ mass, this can permit to verify the principle of lepton universality in the standard model with the accuracy a few times better than the existing one.

The resonance depolarization method [5] for calibration of particles energy in storage rings is based on the measurement of the spin precession frequency $\Omega$ of beam electrons in the guiding magnetic field. The spin precession frequency in an ideal storage ring with flat orbits

$$
\Omega=\omega_{0}\left(1+\gamma \frac{\mu^{\prime}}{\mu_{0}}\right) .
$$

with $\omega_{0}$, the revolution frequency; $\mu^{\prime} / \mu_{0}$, the ratio of the anomalous and normal parts of the magnetic moment; $\gamma$,

\footnotetext{
${ }^{*}$ This work is supported in part by Russian Fund of Basic Research (N 01-02-17477)

† nikitins@inp.nsk.su
}

the relativistic factor to be determined. The revolution frequency is known with a high accuracy - better than $10^{-6}$. The value $\Omega$ is found by the resonance depolarization of the beam electrons. The polarized beam is acted by an external oscillating e.m. field whose frequency is scanned. The polarimeter is needed to fix this frequency at the moment of polarization destruction. The process of forced depolarization is slow enough to provide averaging of the particle energy over many periods of synchrotron oscillations. This allows to find the absolute beam energy with an accuracy much better than the beam energy spread. The experiment may be complicated by the fact that the $\tau$ threshold stands in the vicinity of the intrinsic integer spin resonance $\nu=\gamma \mu^{\prime} / \mu_{0}=4(E=1.763 \mathrm{GeV})$ where depolarizing effect of magnetic field imperfections in the storage ring is strengthened. In our case, this means that the existence of beam polarization and the possibility of its application for energy calibration may be significantly limited in time.

\section{SCENARIO OF THE EXPERIMENT}

A measurement of the $\tau^{+} \tau^{-}$production cross section is performed by the detector KEDR in the region most sensitive to the $\tau$ mass - a few MeVs around the threshold (1.78 $\mathrm{GeV}$ ). For the calibration of energy, the polarized electron beams are injected in the storage ring VEPP-4M from the booster storage ring VEPP-3 (see Fig.1). Radiation polarization of particles in VEPP-3 occurs with the characteristic time $\tau_{p} \approx 20$ minutes at the maximal energy of VEPP-3 $E=2 \mathrm{GeV}$. Before a beam injection in VEPP-4M, the energy in VEPP-3 is lowered to the energy region above the $\tau$ threshold, but below the spin resonanses in VEPP-3, determined by combinations of the spin frequency and betatron tunes. Conservation of beam polarization in the process may be provided with the technique of fast spin resonance crossing [5]. Alternatively, the beam polarization in VEPP3 is organized at the energy of injection. The 3D spin rotations in magnets of the injection line decrease the vertical projection of polarization at VEPP-4M by a few percents. The depolarizing effect of quantum fluctuations in the presence of magnetic imperfections leads to disappearence of the beam polarization in VEPP-4M during the spin relaxation time $\tau_{r}$. The extent of depolarization is given by the factor $G=P / P_{0}=\tau_{r} / \tau_{p}<1$ where $P$ and $\tau_{r}$ stand re- 
spectively for the equilibrium degree and time of radiation polarization in the real machine with imperfections. Corresponding parameters in the ideal machine are $P_{0}=0.92$ and $\tau_{p}$. The value $\tau_{p}$ varies with the energy as $E^{-5}$ and

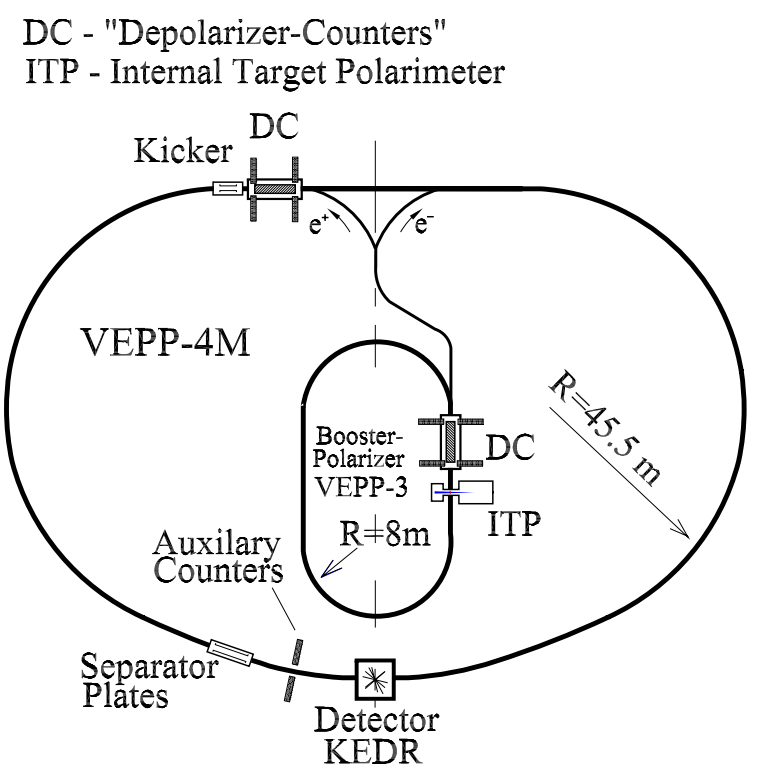

Figure 1: Layout of the polarimeters at VEPP-4.

is quite great near the $\tau$ threshold for VEPP-4M: $\tau_{p} \approx 85$ hours. By this reason, even with $G<<1$, the time $\tau_{r}$ may be comparable with the time of one beam run in the sufficient neighbourhood of the $\tau$ threshold.

\section{DEPOLARIZING EFFECT OF MAGNETIC IMPERFECTIONS}

Parasitic radial magnetic fields in the storage ring is assumed to be the main depolarizing factor in the region of the $\tau$ threshold. Their effect is given by the formula [5]

$$
\frac{\tau_{p}}{\tau_{r}} \approx \frac{11 \pi^{4}}{54} \frac{\nu^{4}\left(1+2 \cos ^{2} \pi \nu\right)}{\sin ^{4} \pi \nu} \sum_{j=1}^{N} \eta_{j}^{2} h_{j}^{2}\left|F_{j}^{\nu}\right|^{2}
$$

where $N$ is the number of perturbations; $\eta_{j}$ is a fraction of the orbit occupied by the $j$-th perturbution; $\eta_{j} h_{j}=\delta z_{j} / f_{j}$ if the radial field is due to the vertical misalignment $\left(\delta z_{j}\right)$ of the quadrupole lens with the focal length $f_{j}$. The complex value $F^{\nu}$ is the spin response function [5] which shows how the direct depolarizing action of the perturbation in the form of transverse e.m. field can be amplified due to vertical betatron oscillations excited by this field. In the machine with strong focusing, the $F^{\nu}$ depends not only on the energy and the vertical betatron tune but also on the general view of magnetic structure [6]. For VEPP-4M $(\delta z \sim 100$ $\mu \mathrm{m}, \bar{f} \approx 330 \mathrm{~cm}, N \sim 100) G=\tau_{r} / \tau_{p} \sim 1 / 150$ at $E=1777 \mathrm{MeV}\left(\nu=4.03, \overline{\left|F^{\nu}\right|^{2}} \approx 12\right)$, i.e. $\tau_{r} \sim 30 \mathrm{~min}-$ utes. The depolarization rate is very strongly varied with the resonance tune $\epsilon=\nu-k: \tau_{r}^{-1} \propto \epsilon^{-4}$.

\section{ON ACCURACY}

The shift and broadening of the spin precession line by virtue of the guiding field non-linearities [5] are estimated to be $\Delta \nu / \nu \leq 10^{-5}$.

The systematical shift of the spin precession frequency due to the influence of radial magnetic fields may be estimated from

$$
\Delta \nu \sim \frac{\nu^{2} \cos \pi \nu}{8 \pi \sin \pi \nu} \sum_{j=1}^{N} \chi_{j}^{2}
$$

where, for the case of vertical closed orbit distortions $z_{0}$ in focusing elements, the perturbation $\chi_{j}=z_{0 j} / f_{j}$. For VEPP-4M this yields $\Delta E / E=\Delta \nu / \nu \sim 5 \cdot 10^{-5}$ at the $\tau$ threshold $(\nu=4.03)$ with $<z_{0}^{2}>^{1 / 2} \approx 1.5 \mathrm{~mm}$.

Inaccurate compensation of the longitudinal magnetic field of KEDR detector by the anti-solenoids gives the spin tune shift

$$
\Delta \nu \approx \frac{\varphi^{2}}{8 \pi} \frac{\cos \pi \nu}{\sin \pi \nu}
$$

where $\varphi$ is the residual angle of velocity rotation around the KEDR axis. Also this defect leads to excitation of the betatron coupling resonance which contributes the frequency split of normal modes by the value $\delta \nu_{C}$. Betatron coupling compensation with anti-solenoids at the level of $\delta \nu_{C} \sim 5 \cdot 10^{-3}$ is quite available and corresponds to the accuracy of $\Delta E / E \sim 1.5 \cdot 10^{-5}$ at $1773 \mathrm{MeV}$.

It is plausible to provide the energy stability at the level of $\sim 10^{-5}$ during the measurement of the $\tau^{+} \tau^{-}$production cross section if the radial deviations in time of the closed orbit $<<1 \mathrm{~mm}$.

\section{POLARIMETERS}

\subsection{Polarimeter based on intra-beam scattering}

The cross section of mutual scattering of electrons (Touschek effect) in the polarized beam is some less than in the unpolarized one. To observe the polarization one needs to depolarize the beam using an external spin resonanse and determing the fact of depolarization by the jump $\propto P^{2}$ in the counting rate of scattered beam particles [5]. Earlier, such a method was used in the measurements of the J/Psiand Psi' mass at VEPP-4 [7]. Magnetic structure of VEPP$4 \mathrm{M}$ and a newly proposed polarimeter scheme differ from that in [7]. The polarimeter device "Depolarizer-Counters" (DC) will be installed in the technical straight sections of both VEPP-4M and VEPP-3 (see Fig.1). It is based on a cylindrical section of vacuum chamber of lengh $830 \mathrm{~mm}$. There is a pair of scintillation counters at the both ends of this section. Counters of each pair can be moved from opposite sides of chamber inward the aperture in the horizontal plane and register the Touschek electrons scattered at the most part of the ring. The two-fold/four-fold coincidence curcuits of counter pulse registration are used to decrease the uncorrelated background influence. To exclude the influence of changes in beam sizes, the closed orbit position as well as the beam lifetime dependence, the method 
of "two bunches" is applied. The value $1-N_{2} / N_{1}$ is under observation where $N_{1}$ and $N_{2}$ are respectively the counting rates of the polarized bunch and the unpolarized one spaced at one-half turn. Numbers of particles in the bunches must be equalized with the accuracy of a few percents. Under the position of counters at a distance of $1 \div 1.5 \mathrm{~cm}$ from the beam, the design counting rate makes up about $10 \mathrm{kHz}$ with the jump of $3 \%$ at the beam current of $\sim 4 \mathrm{~mA}$.

\subsection{Depolarizer}

The two matched striplines with the vertical gap of $60 \mathrm{~mm}$ between them are mounted inside of the polarimeter section to create the TEM wave moving towards the beam. The alternative variant may be the use of matched plates of the separator system at the experimental area of VEPP4M. The signal source is the frequency synthesizer controlled by a computer with the minimal band width of $\Delta f_{d} \sim 1-10 \mathrm{~Hz}$ and the rearrangement step of $1 \mathrm{~Hz}$. The power wide-band amplifier provides the amplitude of the voltage between striplines up to $U_{d} \approx 400$ Volts. The rate $\left(\tau_{d}^{-1}\right)$ of forced depolarization with the transverse field crucially depends on the value $\left|F^{\nu}\right|$ at the place of the depolarizer location: $\tau_{d}^{-1} U_{d}^{2} \propto\left|F^{\nu}\right|^{2} / \Delta f_{d}$. The design depolarization time $\tau_{d}$ is a few tens of seconds at $\mathrm{E}=1780 \mathrm{MeV}$ with $U_{d} \approx 200$ Volts, $\Delta f_{d}=10 \mathrm{~Hz}$. The use of alternative plates may be preferrable to decrease the necessary value $U_{d}$ since the factor $\left|F^{\nu}\right|^{2}$ at the place of their position is about 100 times greater than for striplines placed at the technical area of VEPP-4M.

\subsection{Mфller polarimeter with internal target}

With the aim to check the polarization at VEPP-3, we intend [9] to use a nondestructive method based on watching the asymmetry in scattering of beam electrons by the internal polarized target (ITP) [5]. The effect of azimuthal asymmetry with a transverse polarization of both the beam and the target electrons reaches $1 / 9$. This may be doubled if it is possible to change a sign of the target polarization. To date such a method has not been applied yet in experiments with circulating beams anywhere. Our internal target will be presented by the deuterium jet being injected from the Atom Beam Source (ABS) of the DEUTERON Facility [8] into the vacuum chamber of VEPP-3 (see Fig.1). Electron polarization of the ABS beam is close to unity. A special magnetic system creates the periodically varied in sign field to control the target polarization. Two detectors, positioned above and down with respect to the median plane and removed from the target at the distance optimized to observe the polarization effect, register a pair of the beam electron and the jet one providing their correlation in two transverse coordinates. The design asymmetry in counting rate is expected to be as 5 times as a statistical error in 8 minutes at the beam polarization of $80 \%$ and the beam current of $\sim 100 \mathrm{~mA}$. The preliminary experiment has been performed [9] during the run on measurement of deuteron formfactor at the DEUTERON facility.

\subsection{Test observations at VEPP-4M}

We have tested the polarimeter system based on intra-beam scattering in the energy range of J/Psi peak using two auxiliary scintillation counters mounted on the outside of the vacuum chamber (see Fig.1). In spite of the low counting rate of $\sim 100 \mathrm{~Hz}$ in this case, the accuracy achieved in determination of the depolarization frequencies (see Fig.2) corresponds to the accuracy in energy of $\sim 10^{-5}$. The fact, that it is precisely the spin precession frequency, was sustained by the J/Psi peak position found with KEDR.

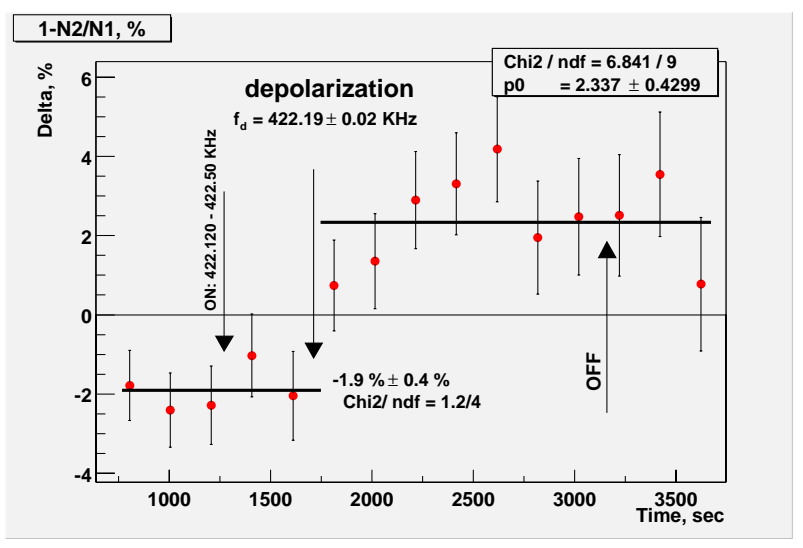

Figure 2: The observation of jump during one of the scanning of depolarizer frequency.

\section{SUMMARY}

The accuracy better than $10^{-4}$ in beam energy calibration at the region above the $\tau$ threshold is quite achievable. Going toward the $\tau$ threshold, the parasitic beam depolarization may noticeably bound the calibration procedure in time.

We thank A.N. Skrinsky, Yu.M. Shatunov and S.I. Serednyakov for useful discussions on the beam polarization measurement methods.

\section{REFERENCES}

[1] Submitted to Nucl.Instr. and Meth., 2001.

[2] V.V. Anashin et al, Proc. of 6th European Particle Accelerator Conf., EPAC'98, Stockholm, 1998, v.1, p.400.

[3] D.E.Groom et al, The European Physical Journal C, v.15, No.1-4 (2000).

[4] J.Z. Bai et al, Phys. Rev., D53, 52(1996).

[5] Ya.S. Derbenev et al, Particle Accelerator, v.8 (1978)

[6] S.A.Nikitin et al, NIM, v.216, pp.317-328, (1983).

[7] A.A. Zholents et al, Phys. Lett., v.96B, No.2, pp. 214-216, (1981).

[8] L.G. Isaeva et al, Proc. of 13th Int. Symp. on High Energy Spin Phys., Protvino, Russia, Word Scientific, p.631 (1998).

[9] S.I. Mishnev et al, Abstract Book of the 14th Intern. Spin Physics Symposium “Spin 2000”, Osaka, Japan, Oct. 2000, pp. 294-295. 